[Agr. Biol. Chem., Vol. 32, No. 11, p. 1337 1340, 1968]

\title{
The Aroma of Cigar Tobacco
}

\author{
Part I. Isolation of 2-Hydroxy-2,6,6-trimethylcyclohexylidene- \\ 1-acetic Acid Lactone (Dihydroactinidiolide) \\ from Ether Extract of Cigar Leaves
}

By Hajime Kaneko and Kiyoko IJICHI

Central Research Institute, Japan Monopoly Corporation, Nishi-shinagawa, Shinagawa-ku, Tokyo

Received May 22, 1968

\begin{abstract}
2-Hydroxy-2,6,6-trimethylcyclohexylidene-1-acetic acid lactone was isolated from the steam distillate of the ether extract of cigar leaves.
\end{abstract}

There have been some reports concerning the chemical compositions of cigar smoke. ${ }^{1 \sim 31}$ In these reports volatile acidic and neutral fractions as well as non-volatile acids were investigated. However, any compound which characterizes the special aroma of cigar tobacco has not been obtained. On the other hand, volatile bases of cigar smoke are rather characteristic. Schmeltz and Stedman isolated pyridine-3-ol from cigar smoke. ${ }^{4}$ Osman and Barson identified 2,4-, 2,5-, 2,6- and 3,5lutidines in cigar smoke. ${ }^{51}$ Although these volatile amines are probably the components of the special aroma of cigar tobacco, they do not possess fragrant odors. The chemical compositions of cigar leaves with respect to their special aroma have not been studied. Our investigations are directed toward throwing light on the chemical compositions of the aroma of cigar tobacco.

The ether extract of cigar leaves has an

1) A. I. Schepartz, Tobacco Science, 4, 12 (1960).

2) I. Schmeltz and W.S. Schlotzhauer, ibid., 5, 92 (1961); idem., ibid., 6, 88 (1962).

3) S. Osman and J. Barson, ibid., 10, 85 (1966); idem., Chem and Ind., 1966, 1699.

4) I. Schmeltz and R. L. Stedman, Chem. and Ind., 1962, 1244.

5) S. Osman and J. Barson, Phytochemistry, 3, 587 (1964). aroma very similar to that of cigar tobacco. Its basic fraction has a rather disagreeable aroma, but the neutral fraction has a fragrant one. In this report is described the isolation of 2-hydroxy-2,6,6-trimethylcyclohexylidene-1acetic acid lactone as a fragrant substance from the steam distillate of the neutral fraction of cigar leaves.

The ether extract of Manila leaves (a representative kind of cigar leaves) was fractionated by the usual methods and the neutral fraction was steam-distilled. The distillate had a characteristic aroma which differed from those of Bright Yellow and Japanese domestic tobacco leaves.

It was then subjected to silicic acid column chromatography (Table I). Fraction-6 in Table I has a peach-like fragrance. A dominant peak (P-A) in the gas chromatogram of this fraction was observed at the retention time of $11.3 \mathrm{~min}$ under the conditions described in Fig. 1. Then, isolation of the component corresponding to this peak was attempted. Fraction-6 was rechromatographed with a silicic acid column. In this case the column was eluted successively with $1: 2$ and $1: 1$ mixtures of ether and $n$-hexane. Each eluate was checked by gas chromatography and the fractions containing P-A were combined 
Table I. Chromatographic Separation of THE STEAM Distiltate of THE NEUTRAL Fraction

Fraction
1
2
3
4
5
6
7
8

4.1

3.2

3.2

2. 6

3.9

4.5

$+.0$
Yield

$9.0 \mathrm{~g}$

Volume of each eluent is 2 liters.

to give a fragrant oil. The oil was then subjected to preparative gas chromatography as shown in Fig. 1. Repeated preparative gas chromatography finally gave pure $\mathrm{P}-\mathrm{A}$ as colorless crystals; mp $43.5 \sim 44.0^{\circ} \mathrm{C}$. The yield was about $0.0002 \%$ of the starting dry leaves.

The elemental and mass spectrometric analyses (molecular ion at $\mathrm{m} / \mathrm{e} 180$ ) of $\mathrm{P}-\mathrm{A}$ gave $\mathrm{C}_{11} \mathrm{H}_{16} \mathrm{O}_{2}$ as the molecular formula. The bands at 1755 and $1625 \mathrm{~cm}^{-1}$ in the infrared spectrum of P-A (Fig. 2) indicated the presence of a $\alpha, \beta$-unsaturated $\gamma$-lactone structure in the molecule. This structure was also sup-

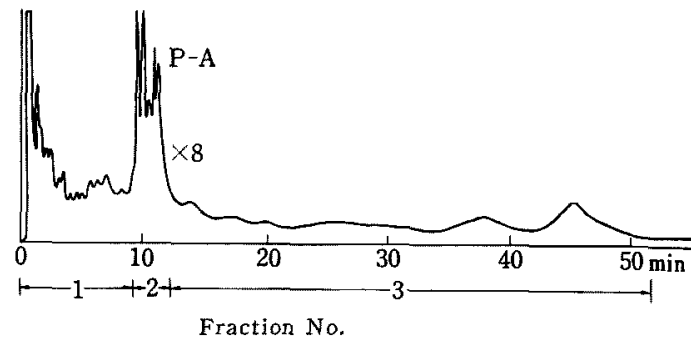

FIG. 1. Preparative Gas Chromatogram of the Fraction Containing P-A.

Conditions; Column: $10 \% 6$ Silicon $\mathrm{SE}-30$ on Diasolid L, $3 \mathrm{~mm} \times 3 \mathrm{~m}$.

Temp.: $190^{\circ} \mathrm{C}$

Carrier Gas: $35 \mathrm{ml} \mathrm{He} / \mathrm{min}$.

ported by the ultraviolet spectrum; $\lambda_{\max }$ $212 \mathrm{~m} \mu, \log \varepsilon$ 4.16. The NMR spectrum of P-A showed the presence of two allylic methyls ( $\delta 1.22$ and 1.28, singlets) and one oleffinic proton ( $\delta 5.52$, singlet). A singlet at $\delta 1.52$ indicated the structure of $\mathrm{CH}_{3}-\mathrm{C}-\mathrm{O}-$. Since the molecular formula showed one more unsaturation except the unsaturated lactone ring, the presence of a saturated aliphatic ring was suggested. From these results, 2-hydroxy2,6,6-trimethylcyclohexylidene-1-acetic acid

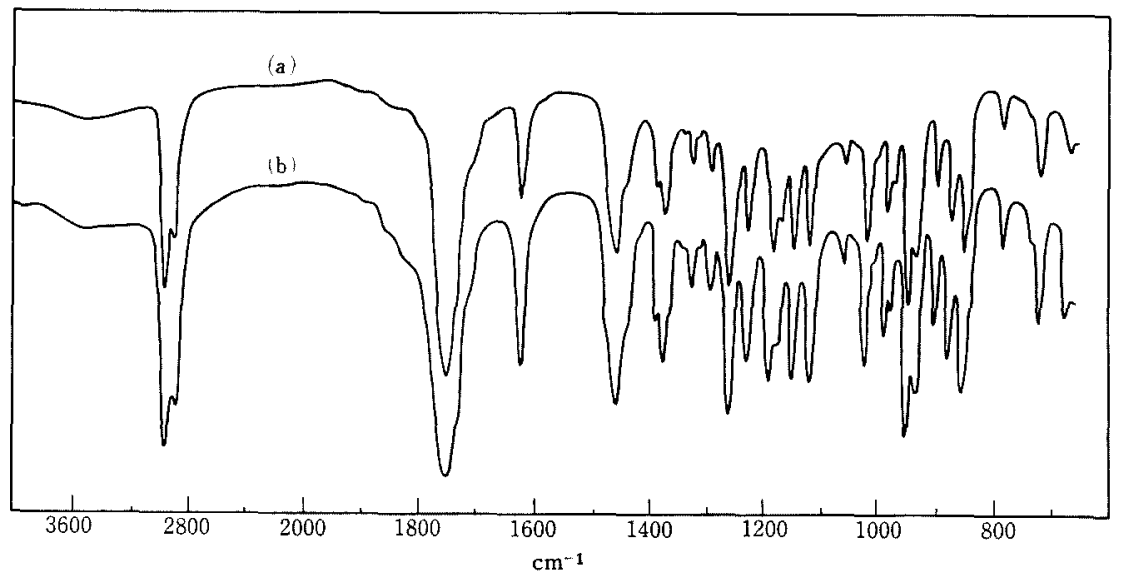

FIG. 2, Comparison of Infrared Spectra.

(a) Sample isolated from cigar tobacco leaves

(b) Synthetic 2-hydroxy-2,6,6-trimethylcyclohexylidene-1-acetic acid lactone

(Each in nujol mull) 
lactone was suggested for the structure of P-A. Recently, Sakan and coworkers isolated a new $\mathrm{C}_{11}$ terpene named "Dihydroactinidiolide" from Actinidia polygama and identified its structure as 2-hydroxy-2,6,6-trimethylcyclohexylidene-1-acetic acid lactone. ${ }^{61}$

To confirm the assumption that P-A is 2hydroxy-2,6,6-trimethylcyclohexylidene-1-acetic acid lactone (I), P-A was compared with the synthetic sample supplied by Dr. Sakan. The infrared spectrum (Fig. 2) and retention time of gas chromatography of P-A were identical with those of the synthetic sample. Although I from Actinidia polygama was reported to have $[\alpha]_{D}+7.1^{\circ}$, the sample derived from tobacco had $[\alpha]_{D}-36.5^{\circ}$ in ethanol and showed slight depression in the mixed melting point (41.0 $43.0^{\circ} \mathrm{C}$ ) with the synthetic sample $(42.0 \sim$ $43.0^{\circ} \mathrm{C}$ ). Pure I from tobacco had a weak pleasant fragrance, but no longer peach-like one. Since the aroma of the fraction which contained P-A varied with the progress of purification, it seems that the aroma of I depends on the presence of a very small amount of impurities and that $I$ acts as an aroma component with some synergetic compounds in tobacco leaves.

Recently, to our great interest, Ina and Sakato isolated I from black tea as an important flavor component. ${ }^{7}$

\section{EXPERIMENTAL}

Infrared measurements were made with a JASCO IR-S spectrophotometer, and the ultraviolet spectrum was measured with a Hitachi ESP-3T in ethanol. The NMR spectrum was measured with a JNMC-100 in carbon tetrachloride, and the mass spectrum with a Hitachi RMU-6D. Specific rotation was measured with a Yanagimoto OR-10 polarimeter. The melting points were uncorrected.

6) T. Sakan, S. Isoe and S. B. Hyeon, Tetrahedron Letters, 1967, 1632.

7) K. Ina and Y. Sakato, Abstracts of Papers, Annual Meeting of the Agricultural Chemical Society of Japan, Nagoya, April, 1968, p. 310; K. Ina, Y. Sakato and H. Fukami, Tetrahedron Letters, 1968 , 2777.
Extraction, fractionation and steam distillation. Twenty-seven kilograms (containing about $8 \%$ moisture) of chopped Manila leaves (Isabela, 1964 crops) were extracted twice with ether and the extract was condensed to about 4 liters by distillation through a Widmer distillation column. Dark green extract thus obtained was successively extracted with $5 \% \mathrm{H}_{2} \mathrm{SO}_{4}$, $5 \% \mathrm{NaHCO}_{3}$ and $5 \% \mathrm{Na}_{2} \mathrm{CO}_{3}$ solutions to obtain basic, acidic and phenolic fractions, respectively. The residual ether layer was washed with water, dried over anhydrous sodium sulfate and distilled through a Widmer distillation column to give $630 \mathrm{~g}$ of the neutral fraction.

The neutral fraction was steam-distilled. About 5 liters of the distillate was collected for each $50 \mathrm{~g}$ of the neutral fraction. The distillate was saturated with $\mathrm{NaCl}$ and extracted with ether. The ether solution was dried over anhydrous sodium sulfate and carefully condensed by distillation through a Widmer column. Thus, $39.5 \mathrm{~g}$ of a characteristic fragrant liquid (containing a small amount of ether) was obtained.

Column chromatography. Silicic acid $(700 \mathrm{~g}$, Mallinckrodt 100 mesh) was mixed with $n$-hexane and the slurry was poured into a column of $10 \mathrm{~cm}$ diameter. To this column ( $15 \mathrm{~cm}$ height) $36.5 \mathrm{~g}$ of the neutral fraction was added. The result of the chromatography was shown in Table I. Fraction-6 in Table I was rechromatographed with a silicic acid column ( $3 \mathrm{~cm} \phi \times 15 \mathrm{~cm}$ height) prepared by the above mentioned method. The column was eluted successively with $300 \mathrm{ml}$ each of $1: 2$ and $1: 1$ mixtures of ether and $n$-hexane, and eluate was collected in each $50 \mathrm{ml}$ fraction. Gas chromatographic analyses showed Fraction-8 and -9 to contain P-A, and both fractions were combined to afford $0.55 \mathrm{~g}$ of a peach-like fragrant oil.

Preparative gas chromatography. The preparative gas chromatography of the above mentioned oil was carıed out with a Shimadzu GC-IB under the same conditions as described in Fig. 1. The temperatures of injection part and detector were 250 and $230^{\circ} \mathrm{C}$, respectively. Five microliters of the above mentioned oil were injected and the effluent was divided into three fractions as shown in Fig. 1. Each effluent was collected into a capillary trap cooled with a dry ice-acetone bath. By repeating the same chromatography and trapping, $40 \mathrm{mg}$ of Fraction-2 in Fig. 1 was obtained from $180 \mathrm{mg}$ of the above mentioned oil. P-A, thus obtained, was a semisolid and seemed to contain some impurities. Further 
purification of this semisolid was carried out by repeating the same preparative gas chromatography as mentioned above to afford $17.5 \mathrm{mg}$ of colorless needles; mp $43.5 \sim 44.0^{\circ} \mathrm{C}$.

Anal. Found: C, 73.25; $\mathrm{H}, 8.83$. Calcd. for $\mathrm{C}_{11} \mathrm{H}_{16} \mathrm{O}_{2}$ : $\mathrm{C}, 73.35 ; \mathrm{H}, 8.93 \% . \quad \lambda_{\max }^{\mathrm{EtOH}} 212 \mathrm{~m} \mu(\log \varepsilon 4.16) . \quad[\alpha]_{\mathrm{D}}^{25}-$ $36.5^{\circ}(c=1.26$ in ethanol).
Acknowledgments. The authors wish to express their thanks to Dr. T. Sakan, Professor of Osaka City University, for his generous gift of synthetic dihydroactinidiolide. Thanks are also due to $\mathrm{Mr}$. K. Aizawa of Tokyo University for NMR measurement and to Hitachi Ltd. for mass spectrometric analysis. 